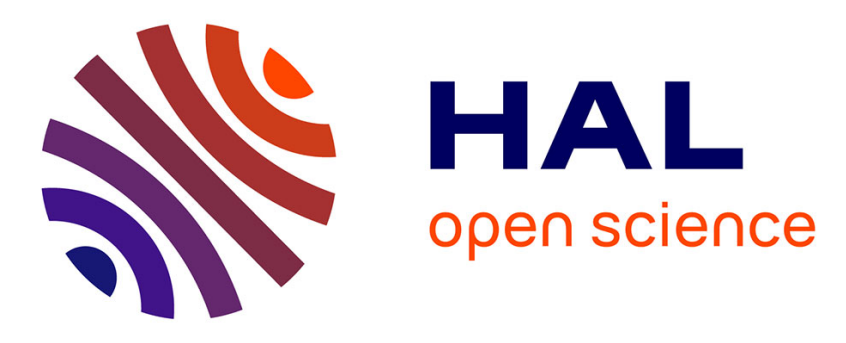

\title{
Fast end efficient single electron transfer between distant quantum dots
}

Sylvain Hermelin, Seigo Takada, M. Yamamoto, Seigo Tarucha, Andreas D.

Wieck, Laurent Saminadayar, Christopher Bäuerle, Tristan Meunier

\section{- To cite this version:}

Sylvain Hermelin, Seigo Takada, M. Yamamoto, Seigo Tarucha, Andreas D. Wieck, et al.. Fast end efficient single electron transfer between distant quantum dots. Journal of Applied Physics, 2013, 113, pp.136508. 10.1063/1.4795528 . hal-00997028

\section{HAL Id: hal-00997028 \\ https://hal.science/hal-00997028}

Submitted on 27 May 2014

HAL is a multi-disciplinary open access archive for the deposit and dissemination of scientific research documents, whether they are published or not. The documents may come from teaching and research institutions in France or abroad, or from public or private research centers.
L'archive ouverte pluridisciplinaire HAL, est destinée au dépôt et à la diffusion de documents scientifiques de niveau recherche, publiés ou non, émanant des établissements d'enseignement et de recherche français ou étrangers, des laboratoires publics ou privés. 


\section{AIP $\mid \begin{aligned} & \text { Juanalo } \\ & \text { Applied Physics }\end{aligned}$}

Fast and efficient single electron transfer between distant quantum dots

S. Hermelin, S. Takada, M. Yamamoto, S. Tarucha, A. D. Wieck et al.

Citation: J. Appl. Phys. 113, 136508 (2013); doi: 10.1063/1.4795528

View online: http://dx.doi.org/10.1063/1.4795528

View Table of Contents: http://jap.aip.org/resource/1/JAPIAU/v113/i13

Published by the American Institute of Physics.

\section{Additional information on J. Appl. Phys.}

Journal Homepage: http://jap.aip.org/

Journal Information: http://jap.aip.org/about/about_the_journal

Top downloads: http://jap.aip.org/features/most_downloaded

Information for Authors: http://jap.aip.org/authors

\section{ADVERTISEMENT}

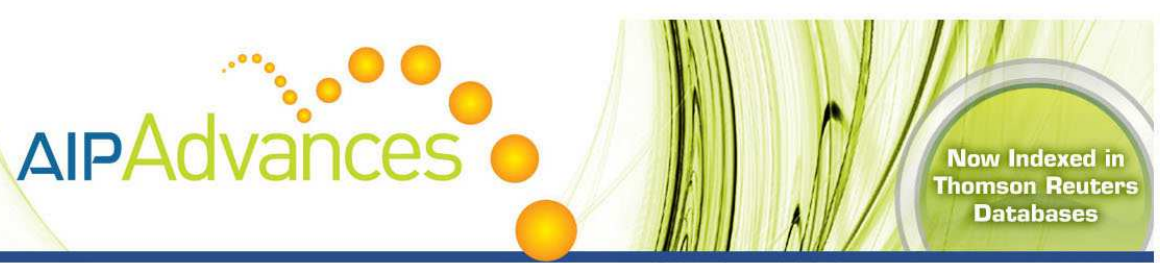

\section{Explore AIP's open access journal: •Rapid publication \\ - Article-level metrics \\ - Post-publication rating and commenting}




\title{
Fast and efficient single electron transfer between distant quantum dots
}

\author{
S. Hermelin, ${ }^{1}$ S. Takada, ${ }^{2}$ M. Yamamoto, ${ }^{3}$ S. Tarucha, ${ }^{4}$ A. D. Wieck, ${ }^{5}$ L. Saminadayar, ${ }^{1}$ \\ C. Bäuerle, ${ }^{1}$ and T. Meunier ${ }^{1}$ \\ ${ }^{1}$ I. Néel, CNRS and Université Joseph Fourier, 25 Rue des Martyrs, 38000 Grenoble, France \\ ${ }^{2}$ Department of Applied Physics, University of Tokyo, Tokyo 113-8656, Japan \\ ${ }^{3}$ ERATO-JST, Kawaguchi-shi, Saitama 331-0012, Japan \\ ${ }^{4}$ ICORP (International Cooperative Research Project) Quantum Spin Information Project, Atsugi-shi, \\ Kanagawa 243-0198, Japan \\ ${ }^{5}$ Lehrstuhl für Angewandte Festkörperphysik, Ruhr-Universität Bochum, Universitätsstrasse 150, \\ 44780 Bochum, Germany
}

(Received 1 October 2012; accepted 3 January 2013; published online 29 March 2013)

\begin{abstract}
Lateral quantum dots are a promising system for quantum information processing devices. The required basic manipulations of a single electron spin have indeed been demonstrated. However, a stringent requirement is the ability to transfer quantum information from place to place within one sample. In this work, we explore and demonstrate the possibility to transfer a single electron between two distant quantum dots in a fast and reliable manner. (C) 2013 American Institute of Physics. [http://dx.doi.org/10.1063/1.4795528]
\end{abstract}

\section{INTRODUCTION}

Lateral quantum dots have seen tremendous improvements over the past years and allow to manipulate a single electron or its spin quantum coherently. ${ }^{1-5}$ In addition, double dots-i.e., two dots lithographied close to each other-offer the possibility to make two electrons interact with each other in a controlled manner. These tools can hence be seen as new building blocks for electronic devices based on single electrons, whether in classical manner or for a quantum computer. However, in both cases, a major ingredient is missing in the previous list: the possibility to transfer the information, that is the transfer of a single electron from one quantum dot towards another distant one in keeping the electron isolated in the structure. In the following, we show that this missing ingredient has been realised thanks to surface-acoustic-wave assisted transport. In the context of quantum computing, displaced electrons can play the role of photons in superconducting qubits and allow to mediate the interaction between distant qubits.

\section{SAMPLE DESCRIPTION}

The structure used for this work is a GaAs/AlGaAs heterostructure that hosts a 2D electron gas (2DEG) at $95 \mathrm{~nm}$ below the surface, with a mobility $1.5 \times 10^{6} \mathrm{~cm}^{2} / \mathrm{Vs}$ and density $-1.35 \times 10^{11} \mathrm{~cm}^{-2}$ at $4 \mathrm{~K}$. The device was realised by standard optical and electron-beam lithography, e-beam metal deposition, and lift-off techniques. A mesa was defined by wet etching using a solution based on sulfuric acid and oxygenated water. The sample is presented in Fig. 1 and is composed of two lateral quantum dots that will serve as a source and receiver of electrons. They are linked by two long gates that allow to fully deplete the 2DEG between the two quantum dots. This is a stringent requirement in order to avoid receiving an electron previously trapped in the $1 \mathrm{D}$-channel defined when no electron is in the source dot. Furthermore, in the view of realizing a quantum computer based on the spin of the electron, it is necessary that the injected electron is the one which is received in the detector since its spin will carry information. It directly follows that we need electrometers in order to determine in a single shot manner the charge state of the quantum dots. This role will be assumed by Quantum Point Contacts (QPCs) juxtaposed to the quantum dots. It has been shown that such devices allow to detect a change of charge of a single electron in a nearby quantum dot. ${ }^{6-8}$ The QPCs were typically biased with $300 \mu \mathrm{eV}$. They allowed to establish the stability diagrams shown in Figure 1, which show that the single electron regime is reached for each dot. ${ }^{9}$ A further tool is needed: If one electron is simply "pushed" into the 1D-channel, it will stay blocked on irregularities of the potential. It is thus necessary to assist it on its journey. This help is brought by Surface Acoustic Waves (SAWs), which will drag the electron along the wafer. SAWs are generated by a 30-nm-thick $\mathrm{TiAu}$ InterDigitated Transducer (IDT) thanks to the piezoelectricity of GaAs. It is composed of 70 pairs of fingers, $60 \mu \mathrm{m}$ in length. The spatial periodicity of the design is $\lambda \simeq 1 \mu \mathrm{m}$. When polarised with an electrical voltage of frequency $f=3 \mathrm{GHz}$, it generates an acoustic wave located within $\lambda$ to the surface. Since the material is piezoelectric, this SAW is accompanied by a sinusoidal electrical potential that can drag electrons in the $2 \mathrm{DEG} .{ }^{10}$ More recent work has shown that when a QPC or 1D-channel is irradiated by such a SAW, it can drag a current through the sample, quantized in units of $e \times f$, where $e$ is the electron charge. ${ }^{11}$ This implies that each minimum of the acoustic wave can be loaded by exactly $0,1,2, \ldots$ electrons. The combination of a SAW and a 1D-channel thus mimics a quantum dot, ${ }^{12}$ that is moving in the $2 \mathrm{DEG}$ at a speed of $3000 \mathrm{~m} / \mathrm{s}$, hence the name of dynamical quantum dot (DyQD). We can hence use one of these DyQDs to transport one electron from one side of the sample to the other through the 1D-channel.

\section{EFFICIENT TRANSFER OF A SINGLE ELECTRON}

Since the 1D-channel is pinched off, its potential floor is higher in energy than the Fermi level of the reservoirs. We 


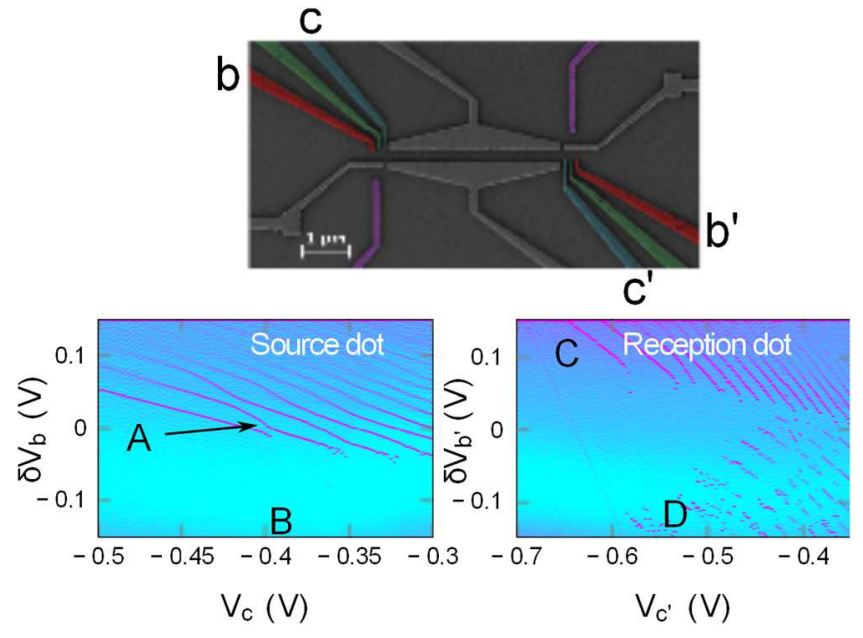

FIG. 1. Top: False colors SEM image of the sample used. Bottom: Stability diagrams of both dots. The zero electron region is reached in the bottom-left corner of the two diagrams.

will then need to push the electrons higher in energy if we want them to be able to reach the 1D-channel (see Fig. 2). Figure 2 shows QPC traces resulting from a gate sequence that loads one electron (in the $n=1$ region of the dot's stability diagram, Fig. 1, A) and then pushes it high in energy into a metastable position ${ }^{13}$ (into the $n=0$ region, Fig. $1, \mathrm{~B}$ ). We can clearly see two levels for the QPC trace: The dot is loaded with 0 or 1 electron. If loaded with one electron on arrival at the metastable point, it leaves the dot after a stochastic time. If averaged, we recover an exponential decay towards 0 electron with a characteristic time around $600 \mathrm{~ms}$, characteristic of the tunneling back to the reservoir. The same experiment can be repeated, but now a burst of acoustic wave is generated $100 \mathrm{~ms}$ after the dot is set in the metastable position. The results indicate (data not shown here) that the electron gets out of the quantum dot with a high probability. In order to check
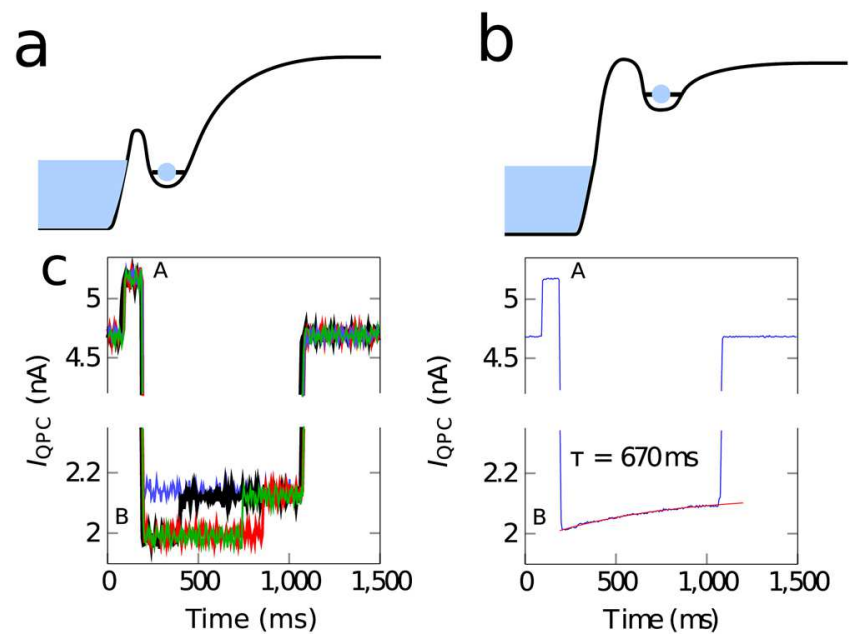

FIG. 2. Schematic view of the electrical potential of the injection dot at load point (a) and at the metastable point (b). (c) Time traces of the injection QPC for the gate sequence describe where an electron is first injected in the dot (position A) and then brought in the metastable position B above the Fermi energy where the electron tunnels out of the dot with timescale approaching $1 \mathrm{~s}$. The loading event (at A) is not resolved, since faster than our measuring electronics. The traces around $4.6 \mathrm{nA}$ correspond to a rest position of the voltage sources. that the electron is indeed transported through the 1D-channel and is not simply kicked back into the reservoir, ${ }^{13}$ we will set up the second quantum dot to try and catch the electron.

The reception quantum dot is thus polarized deep in the metastable region (point $\mathrm{D}$ in Figure 1). This configuration allows to get a high and thick barrier between the reservoir and the dot: the electron will indeed be pushed by the SAW, we hence need this barrier high enough to block it. Once the electron is caught, the dot is polarized at point $\mathrm{C}$ to empty it and reset the experiment. The overall sequence is hence as follows: (1) the injection dot is loaded, (2) the reception dot is emptied and set in reception position, (3) the injection dot is set in the metastable position, (4) the charge states are checked for $50 \mathrm{~ms}$, (5) a burst of SAW is generated, and (6) the (new) charge states are measured for $50 \mathrm{~ms}$. The resulting single shot traces observed experimentally are presented in Figure 3. Statistics are accumulated for 10001 repetitions in different loading situations in the source dot. This analysis allows to completely characterize the transfer process and shows that it is indeed the electron that is loaded in the injection dot that actually arrives in the reception dot with a high efficiency: $95 \%$ for the injection and $92 \%$ for the reception. ${ }^{14}$ This high efficiency and reliability have been confirmed in a simultaneous work by McNeil and co-authors. ${ }^{15}$

\section{NANOSECOND TRIGGERED ELECTRON TRANSFER}

An important parameter for the use of this system in a quantum computer resides in the ability to trigger every
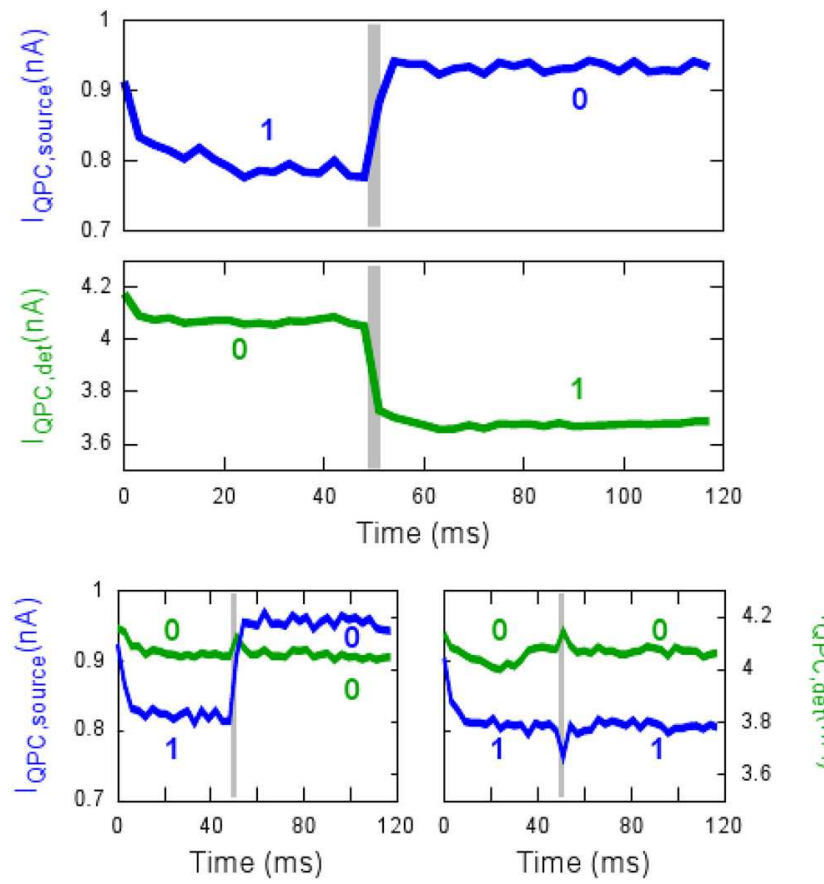

FIG. 3. Upper part: Single shot QPC response as a function of the time recorded during the transfer procedure. The grey line represents the instant at which we apply the microwave burst. For the source dot, the signal jumps from the one-electron conductance to the zero-electron conductance. For the reception dot, the opposite takes place. Lower part: Experimental single shot traces where an error is happening during the transfer. Left: The electron did not reach the reception dot. Right: The electron was not sent during the procedure. 

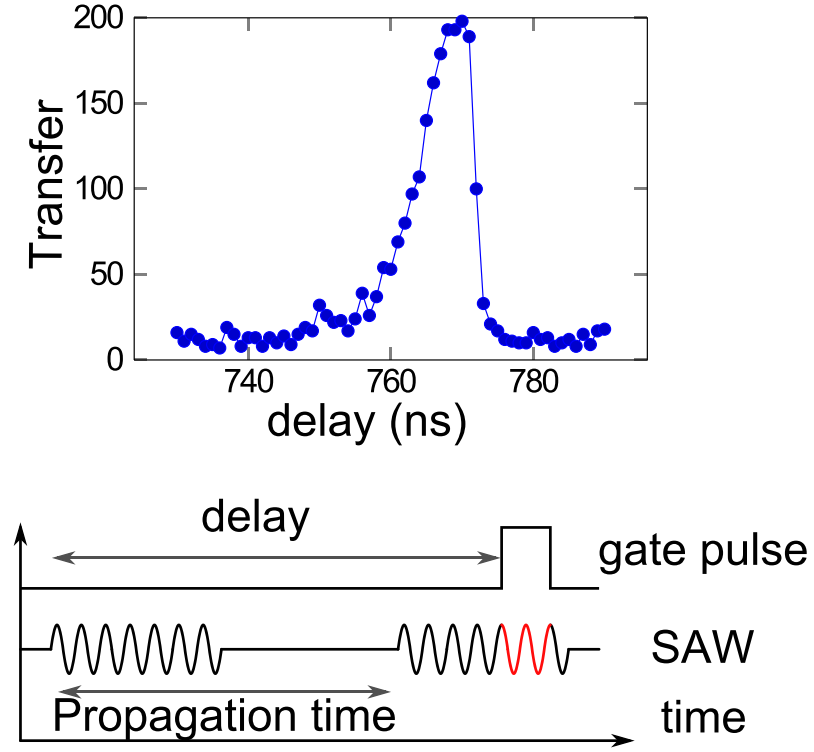

FIG. 4. Evolution of the transfer success number as a function of the delay between the SAW generation and the 1-ns pulse on $V_{C}$, out of 200 repetitions. The SAW duration is $65 \mathrm{~ns}$. Lower panel: Timing schematic of the experiment. The time of flight is the time needed for the SAW to travel from the IDT to the centre of the sample-around $2 \mathrm{~mm}$.

operation faster than the coherence time of a single electron spin. If spin echo sequences can raise this time up to $200 \mu \mathrm{s}$, the "bare" coherence time is typically around $10 \mathrm{~ns} .{ }^{2,16-18} \mathrm{We}$ thus need to trigger the transfer on this timescale. However, the experiments already presented here used a SAW burst of $65 \mathrm{~ns}$, which is the limit imposed by the bandwidth of the IDT. The idea to get triggering at the nanosecond timescale is first to bury the electron deeper in the emission dot so that the SAW excitation is not enough to inject the electron into the moving quantum dots. Second, the addition of a voltage pulse on gate c (see Figure 1) during 1 ns allows to bring back the electron in a situation where it is more sensitive to SAW excitation and allows to turn back on the injection probability (while conserving the good transfer efficiency). The corresponding transfer probabilities are presented in Figure 4, where the delay between the generation of the SAW burst and the ns-gate is varied.

This shows that the injection can indeed be triggered within $1 \mathrm{~ns}$ and the limit to go lower should only be technological (a faster pulse generator with the same amplitude should allow the same result on faster time scales).

\section{CONCLUSION}

In conclusion, we have demonstrated a fast and efficient transfer method for a single electron between two distant quantum dots. Further investigations on the spin of the transported electrons are to be realized to fully characterize the abilities of this system.

${ }^{1}$ J. M. Elzerman, R. Hanson, L. H. Willems van Beveren, B. Witkamp, L. M. K. Vandersypen, and L. P. Kouwenhoven, "Single-shot read-out of an individual electron spin in a quantum dot," Nature 430, 431-435 (2004).

${ }^{2}$ J. R. Petta, A. C. Johnson, J. M. Taylor, E. A. Laird, A. Yacoby, M. D. Lukin, C. M. Marcus, M. P. Hanson, and A. C. Gossard, "Coherent manipulation of coupled electron spins in semiconductor quantum dots," Science 309, 2180-2184 (2005).

${ }^{3}$ F. H. L. Koppens, C. Buizert, K. J. Tielrooij, I. T. Vink, K. C. Nowack, T. Meunier, L. P. Kouwenhoven, and L. M. K. Vandersypen, "Driven coherent oscillations of a single electron spin in a quantum dot," Nature 442, 766-771 (2006).

${ }^{4}$ M. Pioro-Ladrière, T. Obata, Y. Tokura, Y.-S. Shin, T. Kubo, K. Yoshida, T. Taniyama, and S. Tarucha, "Electrically driven single-electron spin resonance in a slanting Zeeman field," Nat. Phys. 4, 776 (2008).

${ }^{5}$ R. Brunner, Y. S. Shin, T. Obata, M. Pioro-Ladrière, T. Kubo, K. Yoshida, T. Taniyama, Y. Tokura, and S. Tarucha, "Two-qubit gate of combined single-spin rotation and interdot spin exchange in a double quantum dot," Phys. Rev. Lett. 107, 146801 (2011).

${ }^{6}$ C. Rössler, S. Baer, E. d. Wiljes, P. L. Ardelt, T. Ihn, K. Ensslin, C. Reichl, and W. Wegscheider, "Transport properties of clean quantum point contacts," New J. Phys. 13, 113006 (2011); e-print arXiv/1106.2982. ${ }^{7}$ D. A. Wharam, T. J. Thornton, R. Newbury, M. Pepper, H. Ahmed, J. E. F. Frost, D. G. Hasko, D. C. Peacock, D. A. Ritchie, and G. A. C. Jones, "Onedimensional transport and the quantisation of the ballistic resistance," J. Phys. C 21, L209 (1988).

${ }^{8}$ M. Field, C. G. Smith, M. Pepper, D. A. Ritchie, J. E. F. Frost, G. A. C. Jones, and D. G. Hasko, "Measurements of Coulomb blockade with a noninvasive voltage probe," Phys. Rev. Lett. 70, 1311-1314 (1993).

${ }^{9}$ R. Hanson, J. R. Petta, S. Tarucha, and L. M. K. Vandersypen, "Spins in few-electron quantum dots," Rev. Mod. Phys. 79, 1217 (2007).

${ }^{10}$ M. J. Hoskins, H. Morkoç, and B. J. Hunsinger, "Charge transport by surface acoustic waves in GaAs," Appl. Phys. Lett. 41, 332-334 (1982).

${ }^{11}$ J. M. Shilton, V. I. Talyanskii, M. Pepper, D. A. Ritchie, J. E. F. Frost, C. J. B. Ford, C. G. Smith, and G. A. C. Jones, "High-frequency singleelectron transport in a quasi-one-dimensional GaAs channel induced by surface acoustic waves," J. Phys.: Condens. Matter 8, L531-L539 (1996).

${ }^{12}$ M. Astley, M. Kataoka, C. Ford, C. Barnes, D. Anderson, G. Jones, I. Farrer, D. Ritchie, and M. Pepper, "Energy-dependent tunneling from few-electron dynamic quantum dots," Phys. Rev. Lett. 99, 156802 (2007).

${ }^{13}$ M. Kataoka, R. J. Schneble, A. L. Thorn, C. H. W. Barnes, C. J. B. Ford, D. Anderson, G. A. C. Jones, I. Farrer, D. A. Ritchie, and M. Pepper, "Single-electron population and depopulation of an isolated quantum dot using a surface-acoustic-wave pulse," Phys. Rev. Lett. 98, 046801 (2007).

${ }^{14}$ S. Hermelin, S. Takada, M. Yamamoto, S. Tarucha, A. D. Wieck, L. Saminadayar, C. Bäuerle, and T. Meunier, "Electrons surfing on a sound wave as a platform for quantum optics with flying electrons." Nature 477, 435-438 (2011).

${ }^{15}$ R. P. G. McNeil, M. Kataoka, C. J. B. Ford, C. H. W. Barnes, D. Anderson, G. A. C. Jones, I. Farrer, and D. A. Ritchie, "On-demand single-electron transfer between distant quantum dots," Nature 477, 439-442 (2011).

${ }^{16}$ I. A. Merkulov, A. L. Efros, and M. Rosen, "Electron spin relaxation by nuclei in semiconductor quantum dots," Phys. Rev. B 65, 205309 (2002).

${ }^{17}$ J. M. Taylor, J. R. Petta, A. C. Johnson, A. Yacoby, C. M. Marcus, and M. D. Lukin, "Relaxation, dephasing, and quantum control of electron spins in double quantum dots," Phys. Rev. B 76, 035315 (2007).

${ }^{18}$ H. Bluhm, S. Foletti, I. Neder, M. Rudner, D. Mahalu, V. Umansky, and A. Yacoby, Nat. Phys. 7, 109-113 (2011). 